\title{
DEXTROSE PROLOTHERAPY VERSUS LOW LEVEL LASER THERAPY (LLLT) FOR MANAGEMENT OF TEMPOROMANDIBULAR JOINT DISORDERS (TMD): CLINICAL RANDOMIZED CONTROLLED STUDY
}

\author{
Nermeen Hassanien*, Heba M. Kamel ${ }^{* *}$ and Sayed A. Rashed ${ }^{* * *}$
}

\begin{abstract}
Objective: To compare the effects of dextrose prolotherapy and low level laser therapy in terms of the pain (VAS) and maximum interincisal opening (MIO) in treatment of temporomandibular disorders.

Patients and Methods: Twenty patients (10 males \& 10 females) with temporomandibular disorders TMD participated in this clinical trial. All patients were randomly divided into two equal groups. In dextrose group; 12.5\% Dextrose was injected into 3 targeted areas, the posterior joint space, the anterior disc attachment and superior attachment of masseter muscle. Dextrose prolotherapy was performed at 2 weeks, and 4 weeks intervals. In laser group; semi conductive (diode) gallium arsenide (Ga As) laser was utilized in this study. The therapeutic LLLT application was performed 3 times per week for four consecutive weeks. The primary outcome measure was the severity of the pain at rest assessed with visual analogue scale $(0-10 \mathrm{~cm})$. The secondary outcome measure was Maximum interincisal opening (MIO). All the evaluations in both groups were done at baseline, 2 weeks, and 4 weeks subsequent to the treatment.
\end{abstract}

Results :The results demonstrated insignificant difference between the means of dextrose prolotherapy and laser groups in terms of VAS (at rest), MIO degree values before treatment (base line) ( $p>0.05)$. When the changes in the groups after treatment were compared, there was no significant difference between the means of dextrose and laser groups regarding VAS (at rest) after 2 weeks and 4 weeks (p- value $>0.05$ ). However, there was a statistical significant difference $(\mathrm{P}$-value $=0.001)$ between the means of both groups regarding MIO after 2 weeks and 4 weeks (P-value $<0.001)$. The improvement in maximum interincisal opening was greater in the group that submit to dextrose prolotherapy in comparison to the laser group $(\mathrm{p}<0.05)$.

Conclusions: In temporomandibular disorders dextrose prolotherapy is more efficient in improving maximum interincisal opening in comparison to low level laser therapy. However, both treatments have the same effect in reducing pain at rest.

KEY WORDS: Temporomandibular disorders, Low level laser therapy, Dextrose prolotherapy

\footnotetext{
* Associate Professor of Oral and Maxillofacial Surgery Department, Faculty of Dentistry, Cairo University

** Lecturer of Oral and Maxillofacial Surgery Department, Faculty of Dentistry, Cairo University

*** Associate Professor of Oral and Maxillofacial Surgery Department, Faculty of Dentistry, Ahram Canadian University
} 


\section{INTRODUCTION}

Temporomandibular Joint Disorders (TMDs) are considered one of the most common causes of pain in the orofacial area. ${ }^{1}$ It's a collective term used to describe a group of signs and symptoms affecting the temporomandibular joint (TMJ), masticatory muscles and the associated structures. These disorders can affect the quality life of the patient. ${ }^{2}$ Up till now there is no standard treatment protocol for patients with anterior disc displacement with reduction (DDWR). This could be due to the complex nature of this joint, in addition to the lack of complete understanding of the etiology and pathogenesis of this disorder. ${ }^{1}$

In the literature, there is a wide variety of treatment modalities of TMDs, including pharmacological, non-surgical and surgical interventions. The main target is to relieve patient's pain in addition to restoring the normal joint function. ${ }^{3-6}$ Usually the first treatment option used is the performing of jaw exercises by a physiotherapist. ${ }^{1}$ If there is no improvement in this kind of treatment, then medical treatment should be initiated. The medications that are most commonly used in the management of TMDs include nonsteroidal anti-inflammatory drugs (NSAIDs), corticosteroids, anti-depressants and antianxiety drugs and muscle relaxants, Also, oral appliances have gained more popularity in the management of TMDs, with various types of occlusal splints. ${ }^{7-11}$ surgical treatment is usually the last resort in the management of TMD, so it is common to look for other alternative treatments. ${ }^{1}$

One of treatments is the Low Level Laser Therapy (LLLT) which is one of the recently used treatment modalities in the physiotherapy field. It is also well known as soft laser therapy. Authors believe that LLLT have biostimulating, regenerative, analgesic and anti-inflammatory effects. ${ }^{12,13}$ Although the analgesic effect mechanism of LLLT is not yet well understood, it has been elucidated that increased pain threshold by alteration of neuronal stimulation and also inhibition of the medullary reflexes have a role. ${ }^{14,15}$ LLLT causes increased local blood flow due to vasodilatation, which has a major role in treating inflammation. It also causes smooth muscle relaxation. Authors contributes the accelerated healing effect of LLLT to these two factors. ${ }^{16}$

Although this technology has been used for a long period of time in the management of pain, its use in the treatment of TMD is still under debate. Some of the published literature have reported that there is there is no significant difference in the relieve in TMJ pain between the two treatment modalities..$^{15,16}$ While others have found that the use of LLLT is superior to placebo therapy. ${ }^{17,18}$

Another well-known treatment modality for the TMD is the prolotherapy technique, which is considered as an alternative for the surgical treatment. This technique is also known as Regenerative Injection Therapy (RIT), Reconstructive Therapy, Nonsurgical Tendon Ligament and Joint Reconstruction and Growth Factor Stimulation Injection. ${ }^{19-21}$ this technique involves the injection of a substance (which is usually a dextrose solution) in the insertions of ligament and tendon to stimulate fibrous tissue proliferation, thus stabilizing and repairing the fibro-osseous junction. ${ }^{21,22}$ Despite the wide use of prolotherapy, it did not achieve yet a full acceptance by the medical community. ${ }^{23}$ The objective of this study was to evaluate the efficacy of the use of dextrose prolotherapy versus the use of LLLT in the management of patients suffering temporomandibular joint disorders

\section{PATIENTS AND METHODS}

Our study was conducted on twenty patients (ten males and ten females) diagnosed with temporomandibular joint disorders. Patients were selected from the outpatient clinic of the Oral and Maxillofacial Surgery Department, Faculty of dentistry, Ahram Canadian University. All patients signed a consent form. After obtaining approval 
from Ahram Canadian University research and ethical committee

The patients were selected according to the following criteria:

\section{Inclusion criteria:}

1- TMJ pain.

2- Sounds during mandibular movements (clicking, pooping).

3- Functional disability.

4- Age range 16-40 years old.

\section{Exclusion criteria:}

1- Patients taking corticosteroids.

2- Patients on previous treatment of TMJ pain (e.g: occlusal splints).

3- Pregnancy.

4- Presence of medical conditions that interfere with treatment, such as cardiac diseases and patients on pace makers.

\section{Study design:}

This study is a randomized clinical trial. Patients were randomly assigned into two equal groups: group (A) and group (B) according to the website (http//www.random.org.eg). Each group was formed of ten patients:

Group A: Each joint received active application of low level laser therapy using Ga-Al-As (GalliumAluminum-Arsenide) diode laser which was applied in 3 sessions per week for four consecutive weeks

Group B: Each joint received dextrose injections, at base line, two-week and four-week intervals.

\section{Preoperative evaluation:}

Preoperative evaluation included history (including history of pain, dysfunction, previous treatment, associated symptoms, emotional stress, past dental history and detailed medical history) and clinical examination. Data for each patient were collected in his or her own questionnaire and examination chart.

\section{TMJ examination:}

\section{A. TMJ function:}

1- Maximal inter-incisal opening (MIO) as measured in mm from maxillary dental midline to mandibular dental midline.

2- The presence of mandibular deviation during mouth opening.

\section{B. TMJ palpation:}

TMJ was examined by palpation via preauricular and meatal approaches to determine:-

1- The presence of TMJ tenderness or pain.

2- The translatory movement of the condyle during different mandibular movements.

3- Joint sounds: the presence or absence of joint sound.

The study program was explained to the patients, who signed informed consent to undergo treatment.

\section{Laser application (Group A):}

The anatomic landmarks were located by asking the patient to open widely to allow drawing of the articular fossa and then to close lightly on the posterior teeth to draw the condyle within the glenoid fossa.

QUANTA C Laser Unit, QUANTA SYSTEM* spa, Italy (Fig. 1) producing semi conductive (diode) gallium arsenide ( $\mathrm{Ga}$ As) laser was utilized in this study. The therapeutic LLLT (wavelength of 980 nanometers, output power of 0.2 Watt, total energy of $12 \mathrm{~J}$ and exposure time 60 seconds) application was achieved using a laser beam delivered through a handheld single laser probe. 
The LLLT application was achieved through light and direct contact of the laser probe on the affected TMJ; anterior, superior, posterior and lateral to the condyle. The laser beam was continuously delivered from the tip of the laser applicator to the target surfaces. The applicator tip was moving in slow circular motion to assure full exposure of the target surfaces to the laser beam. (Fig. 2) The treatment was performed 3 times per week for four consecutive weeks.

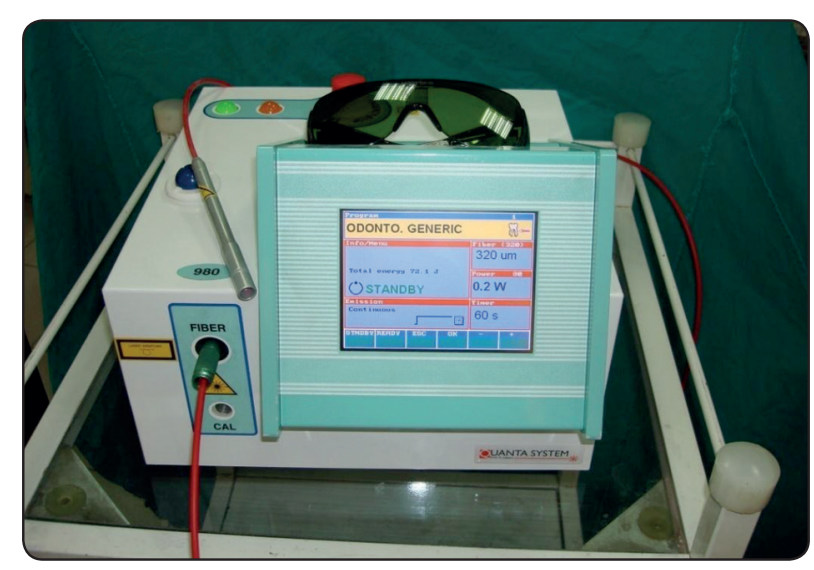

Fig. (1) QUANTA C Laser Unit

\section{Dextrose prolotherapy (Group B):}

The Standard protocol included restriction from non-steroidal anti-inflammatory drugs one to two days before treatment and 10 to 14 days after treatment. After the injection, the patients were cautioned against taking anti-inflammatory agents to relieve the discomfort. After the injection, patients were encouraged to be active and move the injured area.

\section{Prolotherapy technique for temporomandibular disorder (TMD) includes:}

\section{Posture and Head Position:}

The patient was preferably positioned supine or reclined position. Pre-injection procedure and selection of injection tools included the use of one $\mathrm{ml}$ of $25 \%$ Dextrose diluted to $12.5 \%$ by mixing $0.75 \mathrm{~mL}$ of $2 \%$ lidocaine, $0.75 \mathrm{~mL}$ of bacteriostatic water in a $3-\mathrm{mL}$ syringe. A 30 gauge needle of 1 inch length was preferably chosen for the procedure. Before administering injection, the skin over the target area was cleansed with appropriate antiseptic wipes. Then, the target areas were palpated and subsequently marked with a washable felt-tip pen.


Fig. (2) Application of the laser treatment on the affected TMJ; anterior (A), superior (B), posterior (C) and lateral (D) to the condyle. 


\section{The injection Approach:}

The first target area was the posterior joint space which was palpated as the depression that forms immediately anterior to the tragus of the ear as the condyle moves forward and down when the patient opened the mouth. Then, a bite block was placed teeth so that the patient becomes unable to close the mouth during the procedure. The injection needle penetrated the skin at the marked point, directing medially and slightly anteriorly and the needle usually penetrated to nearly its full length before encountering the medial wall of the fossa. Following aspiration, $1 \mathrm{ml}$ of prolotherapy solution was deposited at this site.

The second target was the anterior disc attachment, where the disc connects to the superior head of the lateral pterygoid muscle. This target area was palpated as the slight depression just anterior to the condyle when the mouth is closed. This point was marked before starting injection of targeted areas. The bite block is removed and the patient is instructed to close gently. Then, the needle is inserted at the marked point, directing the tip medially and slightly anteriorly to its full length. Following aspiration, another $1 \mathrm{~mL}$ of prolotherapy solution was injected here.

The attachment of masseter muscle was the next target which was palpated along the inferior border of the zygomatic arch and marked at the same time when the posterior and anterior aspects of the condyle are being evaluated. Asking the patient to clench the teeth, and the area that is most rigid to palpation is usually the tenderest as well. Then, the patient was told to relax and the final one $\mathrm{mL}$ was injected directly into this area. Hence the opposite joint was affected, the head was turned to the other side and the same procedure was repeated on the opposite joint. (Fig. 3)

\section{Postoperative side effects:}

In some cases of PrT, a temporary posterior open bite may result secondary to the introduction of the injection fluid into the articular space. Post injection morbidity may result from a faulty injection technique. some potential complications include discomfort during the procedure, temporary anesthesia extending as far as the eye leading to ptosis, external bleeding, or facial bruising.

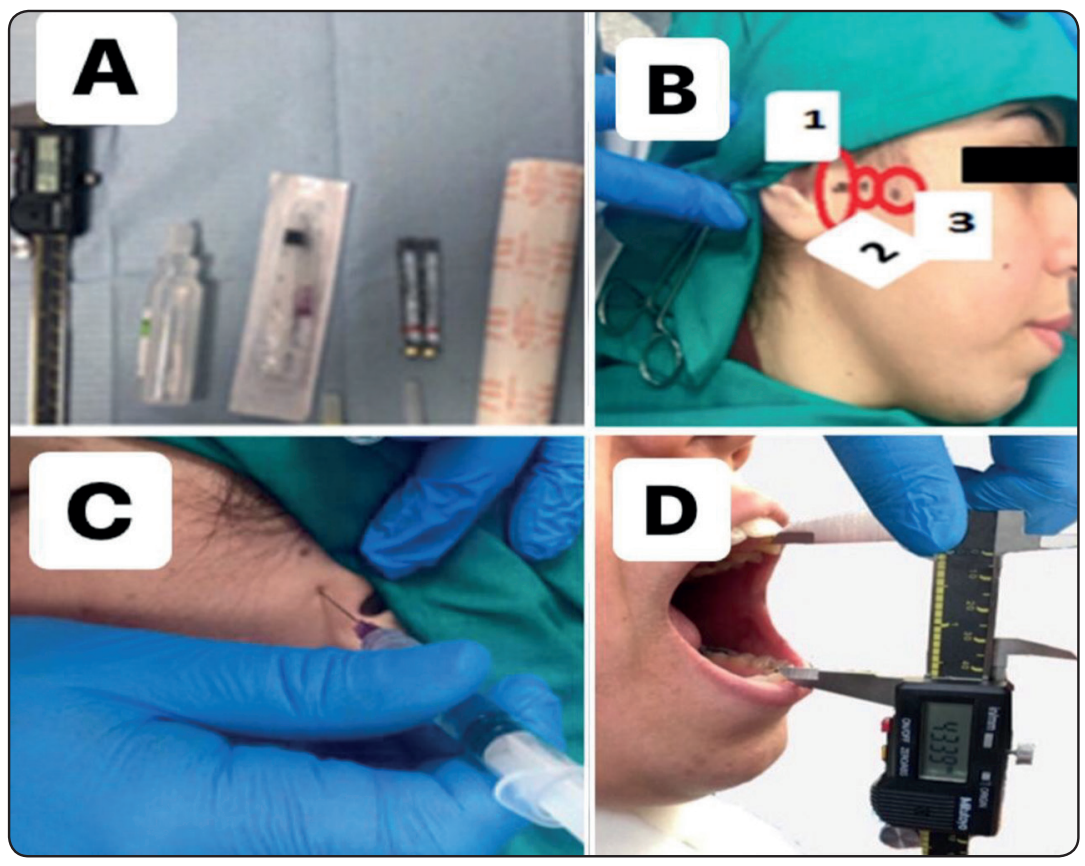

Fig. (3) A. Armamentarium for prolotherapy injection B. 1) the first target area is the posterior joint space. 2) the second target is anterior disc attachment. 3) The Third target is the superior attachment of masseter muscle. C. Injection of posterior joint space D. Postoperative maximal interincisal opening $\mathrm{MIO}$ 
Postoperative assessment: All patients were assessed as follow:

1- Patients' subjective pain experience: Each patient rated his or her pain at rest, using Visual Analog Scale (VAS) of 0-10 with zero being no pain and ten corresponds to the worst pain that the patient ever had.

2- Maximum Interincisal opening (MIO): Assessment of MIO was performed by measuring the distance in $\mathrm{mm}$ between the incisal edges of the upper and lower central incisors using Vernier Caliper.

These parameters (VAS \& MIO) were assessed in both groups at base line, 2 weeks, and 4 weeks postoperative.

\section{Statistical analysis:}

- Microsoft excel 2013was used for data entry and the statistical package for social science (SPSS version 24) was used for data analysis.

- Simple descriptive statistics (arithmetic mean and standard deviation) used for summary of normal quantitative data and frequencies used for qualitative data.
- Independent T-test and Paired T-test was used to compare normally distributed quantitative data among both groups.

- Pearson correlation was used to compare normally distributed quantitative data.

- The level of significance was set at probability (P) value $<0.05$.

\section{RESULTS}

This study was performed on twenty patients (ten males and ten females). The age ranged from 16 to 40 years. Mean age of patients was $26 \pm 4$ years with equal proportions of males and females. About two thirds suffered from pain \& clicking sound $(62.5 \%)$ and almost one third suffered from pain \&tenderness $(37.5 \%)$. Sixty two percent suffered from Internal Derangement and only (37.5\%) from MPD. (Table 1)

Clinical comparisons of both groups were given in Table (2) and figure (4). There was no significant difference between the means of dextrose and laser groups in terms of VAS (at rest) (dextrose 5.88. 2.357 , laser $4.38 \pm 1.506$ ) and MIO degree values before treatment (at base line)(dextrose $35.213 \pm 3.7760$,



Table (1) Demographic features of patients

\begin{tabular}{|c|c|c|}
\hline \multicolumn{2}{|c|}{} & Percent \\
\hline \multirow{2}{*}{ Gender } & Male & $50.0 \%$ \\
\cline { 2 - 3 } & Female & $50.0 \%$ \\
\hline \multirow{2}{*}{ Signs and symptoms } & Pain \& Clicking sound & $62.5 \%$ \\
\cline { 2 - 3 } & pain \&tenderness & $37.5 \%$ \\
\hline \multirow{2}{*}{ Diagnosis } & Internal Derangement & $62.5 \%$ \\
\cline { 2 - 3 } & MPD & $37.5 \%$ \\
\hline
\end{tabular}

\begin{tabular}{|c|c|c|c|c|c|c|c|}
\hline & Mean & Median & Minimum & Maximum & Standard Deviation & Percentile 25 & Percentile 75 \\
\hline Age & 26 & 26 & 21 & 34 & 4 & 23 & 29 \\
\hline
\end{tabular}


There was no significant difference between the means of dextrose and laser groups regarding VAS (at rest) at 2 weeks (Dextrose 3.75 \pm 1.581 and Laser 4.38 \pm 2.066 ) and also regarding VAS at 4

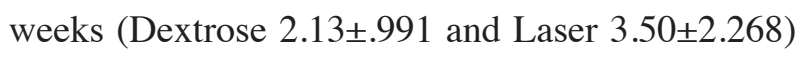
(p- value $>0.05$ ).
In the comparison between both groups Dextrose and Laser one, there was a statistical significant difference $(\mathrm{P}$-value $=0.001)$ between the means of both groups regarding $\mathrm{MIO}$ at 2 weeks (Dextrose $39.4 \pm 2.7$ and Laser 35.2 \pm 1.2 ) and also regarding $\mathrm{MIO}$ at 4 weeks (Dextrose $43.3 \pm 1.7$ and Laser 37.3 \pm 1.9 ) (P-value $<0.001)$. (Table 2, fig. 4)

TABLE (2) Comparison of pain at rest and maximum interincisal opening (MIO) between dextrose and laser groups before treatment, 2 weeks and 4 weeks after treatment. Values are significant when $p$ value $<0.05$ and insignificant when $\mathrm{p}$ value $>0.05$

\begin{tabular}{|c|c|c|c|c|}
\hline & Group & Mean & Std. Deviation & $\mathrm{P}$ value \\
\hline \multirow[t]{2}{*}{ VAS (base line) } & Dextrose group & 5.88 & 2.357 & \multirow[t]{2}{*}{0.152} \\
\hline & Laser group & 4.38 & 1.506 & \\
\hline \multirow[t]{2}{*}{ MIO (base line) } & Dextrose group & 35.213 & 3.7760 & \multirow[t]{2}{*}{0.109} \\
\hline & Laser group & 32.750 & .4629 & \\
\hline \multirow[t]{2}{*}{ VAS (2 ws) } & Dextrose group & 3.75 & 1.581 & \multirow[t]{2}{*}{0.508} \\
\hline & Laser group & 4.38 & 2.066 & \\
\hline \multirow[t]{2}{*}{ MIO (2 ws) } & Dextrose group & 39.4875 & 2.71316 & \multirow[t]{2}{*}{0.001} \\
\hline & Laser group & 35.2500 & 1.28174 & \\
\hline \multirow[t]{2}{*}{ VAS (4 ws) } & Dextrose group & 2.13 & .991 & \multirow[t]{2}{*}{0.138} \\
\hline & Laser group & 3.50 & 2.268 & \\
\hline \multirow[t]{2}{*}{ MIO (4 ws) } & Dextrose group & 43.375 & 1.7069 & \multirow[t]{2}{*}{$<0.001$} \\
\hline & Laser group & 37.375 & 1.9226 & \\
\hline
\end{tabular}

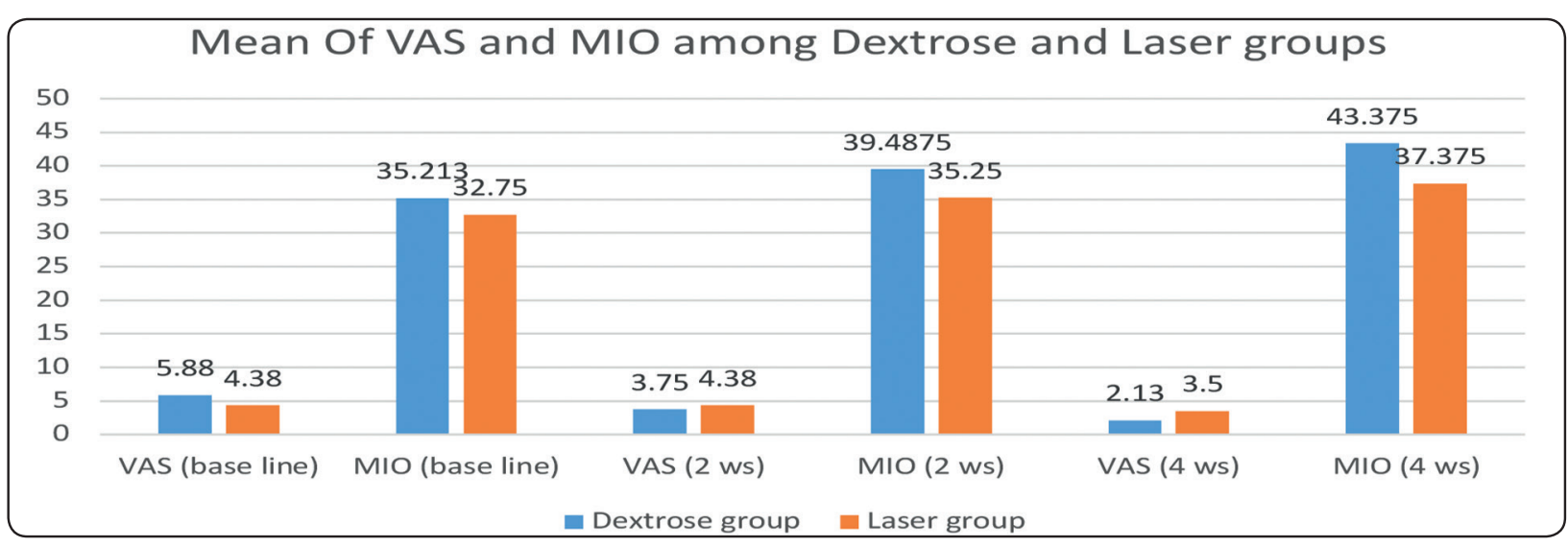

Fig. (4) Shows comparison of pain at rest and maximum interincisal opening (MIO) between dextrose and laser groups before treatment, 2 weeks and 4 weeks after treatment 
Regarding VAS in Dextrose group; Paired Samples Statistics showed that, there was significant difference between the means of VAS before treatment and after 2 weeks (base line 5.88 \pm 2.357 , after 2 weeks $3.75 \pm 1.581)$ ( $\mathrm{P}$ - value $==0.003$ ). There was significant difference between the means of VAS before treatment and 4 weeks (base line $5.88 \pm 2.357$, after 4 weeks $2.13 \pm .991)$ (P-value $=$ $0.004)$. There was significant difference between the means of VAS after 2 and 4 weeks of treatment (2 weeks3.75 $\pm .1 .581,4$ weeks $2.13 \pm 991)$ (P value $=0.019)$. (Table 3$)$.

TABLE (3) Shows the paired wise comparison of VAS at base line, 2 weeks and 4 weeks and also of MIO at base line, 2 weeks and 4 weeks among the dextrose group with statistical significant difference (P-value $<0.005)$ all through the comparisons. Values are significant when $\mathrm{p}$ value $<0.05$ and insignificant when $\mathrm{p}$ value $>0.05$

\begin{tabular}{|l|c|c|c|c|}
\hline \multicolumn{2}{|c|}{} & Mean & $\begin{array}{c}\text { Std. } \\
\text { Deviation }\end{array}$ & \multirow{2}{*}{ P value } \\
\hline \multirow{2}{*}{ Pair 1 } & VAS (base line) & 5.88 & 2.357 & \multirow{2}{*}{0.003} \\
\cline { 2 - 4 } & VAS (2 ws) & 3.75 & 1.581 & \\
\hline \multirow{2}{*}{ Pair 2 } & VAS (base line) & 5.88 & 2.357 & \multirow{2}{*}{0.004} \\
\cline { 2 - 4 } & VAS (4 ws) & 2.13 & .991 & \\
\hline \multirow{2}{*}{ Pair 3 } & VAS (2 ws) & 3.75 & 1.581 & \multirow{2}{*}{0.019} \\
\cline { 2 - 4 } & VAS (4 ws) & 2.13 & .991 & \multirow{2}{*}{0.001} \\
\hline \multirow{2}{*}{ Pair 4 } & MIO (base line) & 35.213 & 3.7760 & \multirow{2}{*}{$<0.001$} \\
\cline { 2 - 4 } & MIO (2 ws) & 39.4875 & 2.71316 & \multirow{2}{*}{$<0.001$} \\
\hline \multirow{2}{*}{ Pair 5 } & MIO (base line) & 35.213 & 3.7760 & \\
\cline { 2 - 4 } & MIO (4 ws) & 43.375 & 1.7069 & \\
\hline \multirow{2}{*}{ Pair 6 } & MIO (2 ws) & 39.4875 & 2.71316 & \multicolumn{2}{|c|}{} \\
\cline { 2 - 4 } & MIO (4 ws) & 43.375 & 1.7069 & \\
\hline
\end{tabular}

Regarding MIO in Dextrose group, Paired Samples Statistics showed that, there was significant difference between the means of MIO before treatment and after 2 weeks (base line $35.213 \pm 3.7760,2$ weeks $39.4875 \pm 2.71316)$ (P value $=0.001$ ). There was significant difference between the means of MIO before treatment and 4 weeks (base line 35.213 \pm 3.776 , 4 weeks $43.375 \pm 1.7069) \quad(\mathrm{P}$ value $=<0.001)$. There was significant difference between the means of MIO after 2 weeks and 4 weeks ( 2 weeks $39.4875 \pm 2.71316,4$ weeks 43.375 \pm 1.7069$)(\mathrm{P}$ value $=<0.001)($ Table 3$)$

Regarding VAS in Laser group; Paired Samples Statistics showed that, there was insignificant difference between the means of VAS before treatment and after 2 weeks ( base line 4.38 \pm 1.506 , after 2 weeks $4.38 \pm 2.066)(\mathrm{P}$ - value=1.000). There was insignificant difference between the means of VAS before treatment and 4 weeks (base line $4.38 \pm 1.506$, after 4 weeks 3.50 $\pm .2 .268)$ (P-value $=$ $0.262)$. There was significant difference between the means of VAS after 2 and 4 weeks of treatment (2 weeks $4.38 \pm .2 .066,4$ weeks $3.50 \pm 2.268)$ (P value $=0.006)$. (Table 4).

Regarding MIO in Laser group, Paired Samples Statistics showed that, there was significant difference between the means of MIO before treatment and after 2 weeks ( base line 32.750 $.4629,2$ weeks $35.2500 \pm 1.28174)$ (P value $=0.004)$. There was significant difference between the means of MIO before treatment and 4 weeks (base line 32.750 \pm .4629 , 4 weeks $37.375 \pm 1.9226)$ ( $\mathrm{P}$ value $=0.001)$. There was significant difference between the means of MIO, 2 weeks and 4 weeks after treatment (2 weeks $35.2500 \pm 1.28174$, 4 weeks $37.375 \pm 1.9226$ ) (P value $=0.013)($ Table 4) 
TABLE (4) Shows the paired wise comparison of VAS at base line, 2 weeks and 4 weeks and also of MIO at base line, 2 weeks and 4 weeks among the laser group with statistical significant difference (P-value $<0.005)$ all through the comparisons except between VAS at base line and 2 weeks,VAS at base line and 4 weeks. Values are significant when $\mathrm{p}$ value $<0.05$ and insignificant when $\mathrm{p}$ value $>0.05$

\begin{tabular}{|c|c|c|c|c|}
\hline & & Mean & $\begin{array}{c}\text { Std. } \\
\text { Deviation }\end{array}$ & $P$ value \\
\hline \multirow{2}{*}{ Pair 1} & VAS (base line) & 4.38 & 1.506 & \multirow{2}{*}{1.000} \\
\hline & VAS (2 ws) & 4.38 & 2.066 & \\
\hline \multirow{2}{*}{ Pair 2} & VAS (base line) & 4.38 & 1.506 & \multirow{2}{*}{0.262} \\
\hline & VAS (4 ws) & 3.50 & 2.268 & \\
\hline \multirow{2}{*}{ Pair 3} & VAS (2 ws) & 4.38 & 2.066 & \multirow{2}{*}{0.006} \\
\hline & VAS (4 ws) & 3.50 & 2.268 & \\
\hline \multirow{2}{*}{ Pair 4} & MIO (base line) & 32.750 & .4629 & \multirow{2}{*}{0.004} \\
\hline & MIO (2 ws) & 35.2500 & 1.28174 & \\
\hline \multirow{2}{*}{ Pair 5} & MIO (base line) & 32.750 & .4629 & \multirow{2}{*}{0.001} \\
\hline & MIO (4 ws) & 37.375 & 1.9226 & \\
\hline \multirow{2}{*}{ Pair 6} & MIO (2 ws) & 35.2500 & 1.28174 & \multirow{2}{*}{0.013} \\
\hline & MIO (4 ws) & 37.375 & 1.9226 & \\
\hline
\end{tabular}

\section{DISCUSSION}

The aim of this study is to compare efficacy of temporomandibular joint prolotherapy and Low Level Laser Therapy (LLLT) that not reduce pain only but improve quality of life. Weakening of the TMJ capsule and ligament that caused by overstretched ligaments would elucidate a lot of the various pathology involving TMD including joint subluxation, disc displacement, as well as muscle spasm and myofascial pain patterns. In the current study, there is no significant difference between the means of dextrose and laser groups regarding VAS (on rest). Dextrose group showed significant decrease in pain scores at rest thought out study while laser group showed significant decrease in pain score at 4 weeks interval, pain scores were significantly decreased in both groups' at 4 weeks. This means that both line of treatment are effective for pain control of temporomandibular disorders. It is detected that dextrose prolotherapy showed more improvements regarding pain score although there is no significant superiority. Dextrose prolotherapy showed pain relief early while laser therapy showed pain relief in intermediate or late phase of therapy. Findings contradict those of other studies $(24,25)$ that reported that laser application could be resulted in the immediate decrease of painful symptoms in the treated group. Improvement in the mandibular movements was observed in both groups throughout the study intervals. In the comparison between both groups Dextrose and Laser groups, there was a statistical significant difference (P-value $=0.001$, $<0.001$ ) between the means of both groups regarding MIO at 2 weeks and at 4 weeks respectively.

Pain medication, splint, physiotherapy and other modalities inducing temporary help and rarely conditions may be cured. Mainly cause of TMD and muscle spasm is under laying ligament laxity. Ligament and capsular repair that induced by prolotherapy could represent more permanent solution. The ligaments of fibrous joint capsule and unite the articular bones, these ligaments provide disc stability. In treatment we need to strengthen and repair the injured capsule and ligament structures. Prolotherapy treatment would have a lasting effect.

Our treatment was injecting dextrose in certain points in temporomandibular joint that contains fibro-osseous junction of the ligament and capsular attachments. We select dextrose due to it is the most common proliferate used in prolotherapy, is readily available, inexpensive, high safety profile and ability to improve stability of the temporomandibular joint by enhancing capsular and ligament strength. The main component of dextrose is glucose. 
When injection done elevation of glucose level and also elevates growth factor level that we need to repair process .Dextrose concentrations that has been used $12.5 \%$, so as it is strongly hypertonic causes cell wall lysis to attract fibroblasts and begin the regenerative process. In temporomandibular joint prolotherapy studies there were clinical improvement after dextrose $12.5 \%$, injection. Results of our study indicate that improvement and tightening of loose ligaments by injection of dextrose $12.5 \%$ that are in agreement with Roy Hakala et al ${ }^{26}$, Jeelani et $\mathrm{al}^{27}$ and Vankdoth et $\mathrm{al}^{28}$. In our study a series of injections, after 2 weeks and then 4 weeks interval have been carried out. Prolotherapy involves the injection of a small amount of proliferative into multiple painful ligament and tendon insertions, trigger points, as well as into the joint spaces to induce healing of the rupured structures. Series of injections 2 to 3 injections was required to often permanently stop the clicking and painful symptoms.

In present research, after the serious of injection as mentioned before we found that there is increase in $\mathrm{MIO}$, enhanced pain scale scores and improvement of clicking. Dextrose induces inflammation that initiates the biological process of wound healing. Following injury, the granulocytes, monocytes and macrophages migrate into the injured tissue. The growth factor is released and activates the fibroblasts, which produce the matrix and new collagen fibrils that lead to tightening of loose ligaments.

The analgesic effect of LLLT could be explained based on the findings of experimental and clinical studies. Our findings are consistent with results of Maia et al ${ }^{29}$ who investigated the effect of LLLT on the pain levels in individuals with TMD in a systematic review. Honmura et al ${ }^{30}$ found that low-power laser irradiation on inflamed regions of rats has a marked analgesic and anti-inflammatory effects. Also, it was found that low-power laser inhibits the increase of vascular permeability, edema in experimentally induced inflammation in rats after receiving laser irradiation. Mazzetto et al ${ }^{31}$ concluded that low intensity laser LLL is an effective modality for the pain control of TMD.

The improvement in mandibular movements in the group treated with laser could not be attributed to its recognized analgesic effect as claimed by Núñez et $\mathrm{al}^{32}$. The observed improvement in mandibular movements (MIO) in the current study is in accordance with those of other studies ${ }^{33-35}$. Variability within individuals might be reflect the effect of psychological factors, such as the desire to feel better which may have influenced physiological processes thereby resulting in the desired outcome. Our findings suggested that dextrose is $t$ better than LLLT at reducing TMJ pain on rest and statistically significant improvement of mandibular movement (MIO).

\section{CONCLUSION}

In the present study, comparison between the laser and dextrose groups did not result in significant difference in pain scores on rest throughout the study intervals. Also, there were significant differences between the two groups in the improvement in the mandibular movements (MIO). Prolotherapy has emerged as an effective treatment with reasonable cost for chronic musculoskeletal and arthritic pain. Clinical improvement of patients with TMJ pain and dysfunction was achieved with both treatment modalities. The limitation of the present study is reduced sample size. However, further studies with larger sample size and longer follow-up periods are necessary to draw decisive conclusions.

\section{REFERENCES}

1. Vijay Kumar A, Jaishankar H, Kavitha A, and Purnachandra Rao Naik. Prolotherapy: A new hope for temporomandibular joint pain. Indian Journal of Pain, 2: 49,2013.

2. Roy V. Hakala and Kim M. Ledermann. The use of prolotherapy in for temporomandibular joint dysfunction. J Prolotherapy, 3: 439, 2010. 
3. Fahimeh Rezazadeh, Khadijeh Hajian, Shoaleh Shahidi, Soraya Piroozi.Comparison of the effects of transcutaneous electrical nerve stimulation and low-level laser therapy on drug-resistent temporomandibular disorders. J Dent Shiraz Uni Med Sci., 18(3): 187-192, 2017.

4. Butts R, Dunning J, Pavkovich R, Mettille J, Mourad F. Conservative management of temporomandibular dysfunction: A literature review with implications for clinical practice guidelines (Narrative review part 2) . J Bodyw Mov Ther, 21(3):541-548, 2017.

5. Armijo-Olivo S, Pitance L, Singh V, Neto F, Thie N, Michelotti A. Effectiveness of Manual Therapy and Therapeutic Exercise for Temporomandibular Disorders: Systematic Review and Meta-Analysis. Phys Ther, 96(1):9-25, 2016.

6. Sumit Bhateja, and Geetika Arora. A Healing Therapy"PROLOTHERAPY" in Dentistry. IJSRST, 3, 2395, 2016.

7. Clark GT, Adachi NY, Dorman MR. Physical medicine procedures affect temporomandibular disorders: A review. J Am Dent Assoc, 119: 121-151, 1990.

8. Medlicott MS and Harris SR. A systematic review of the effectiveness of exercise, manual therapy, electrotherapy, relaxation training, and biofeedback in the management of temporomandibular disorder. Phys Ther, 86: 955-973, 2006.

9. Zhang C, Wu JY, Deng DL, He BY, Tao Y, Niu YM,, Deng MH. Efficacy of splint therapy for the management of temporomandibular disorders: a meta-analysis. Oncotarget, 7(51): 84043-84053, 2016.

10. Ouanounou A, Goldberg M, Haas DA. Pharmacotherapy in Temporomandibular Disorders: A Review. J Can Dent Assoc, Jul;83:h7, 2017.

11. Devi J, Verma M, Gupta R. Assessment of treatment response to splint therapy and evaluation of TMJ function using joint vibration analysis in patients exhibiting TMJ disc displacement with reduction: A clinical study. Indian J Dent Res, 28(1):33-43. 2017.

12. Walsh LJ. The current status of laser applications in dentistry. J Aust Dent, 48:146-155, 2003.

13. Yu W, Naim JO and Lanzafame RJ. The effect of laser irradiation on the release of bFGF from 3 T3 fibroblasts. Photochem Photobiol (1994) 59:167-170, 1994.

14. Sandford Ma and Walsh LJ. Thermal effects during desensitization of teeth with gallium-aluminium-arsenide lasers. Period 15:25-30, 1994.
15. De Abreu Venancio, Camparis CM and Defa'tima Zanirato Lizarelli R. Low intensity laser therapy in the treatment of temporomandibular disorders: a double-blind study. J Oral Rehabil, 32:800-807, 2005.

16. Magri LV, Carvalho VA, Rodrigues FCC, Bataglion C, Leite-Panissi CR. Non-specific effects and clusters of women with painful TMD responders and non-responders to LLLT: double-blind randomized clinical trial. Lasers Med Sci. 33(2):385-392, 2018.

17. Carrasco TG1, Mazzetto MO, Mazzetto RG, Mestriner W Jr. Low intensity laser therapy in temporomandibular disorder: a phase II double-blind study. Cranio, 26(4):274$81,2008$.

18. Bertolucci LE and Grey T. Clinical analysis of mid-laser versus placebo treatment of arthralgic TMJ degenerative joints. J Craniomand Pract, 13:26-29, 1995.

19. Alderman D. Prolotherapy for musculoskeletal pain. Pract Pain Manage, 1:10, 2007.

20. Hauser RA. The Regeneration of articular cartilage with Prolotherapy. J Prolother. 1:39-44, 2009.

21. Fullerton BD. High-resolution ultrasound and magnetic resonance imaging to document tissue repair after prolotherapy: A report of 3 cases. Arch Phys Med Rehabil, 89:377-85, 2008.

22. Ross A. Hauser,Marion A. Hauser, Nicole M. Baird. Evidance Based Use of Dextrose Prolotherapy for Musculoskeletal Pain: A Scientific Literature Review. J. of Prolotherapy, 4: 765, 2011.

23. Mohammad Alsehimya, Ayman Al-Dharrabb and Basem Jamala.The use of dextrose prolotherapy for myofascial pain dysfunction syndrome: a double-blind placebocontrolled study. Egyptian Journal of Oral \& Maxillofac Surg, 3:75, 2016.

24. Mazzetto MO, Hotta TH and de Andrade Pizzo RC. Measurements of Jaw Movements and TMJ Pain Intensity in Patients Treated with GaAlAs Laser. Braz Dent J 21: 356-360, 2010.

25. Mazzetto MO, Carrasco TG, Bidinelo EF, de Andrade Pizzo RC, and Mazzetto RG. Low intensity laser application in temporomandibular disorders: a phase I double-blind study. Cranio 25:186-192, 2007.

26. Roy Hakala ,Kim M , Ledermann : The use of Prolotherapy for Temporomandibular joint Dysfunction ,Journal of Indian Academy of oral medicine \&radiology ; $30,2018$. 
27. Jeelani S, KrishnaS,Reddy J, Reddy V:Prolotherapy in Temporomandibular Disorders: an Overview, Open Journal of Dentistry and Oral Medicine;13189: 15-18, 2013.

28. Vankdoth S, Reddy AS, Talla H, Vijayalaxmi N, Madhulatha G. Prolotherapy - Aventuring treatmentfor temporomandibular joint disorder. IJSS Case Reports \& Reviews; 1(7): 27-30, 2014. Ross A, Marion A, Nicole M: Evidence-Based Use of Dextrose Prolotherapy for Musculoskeletal Pain, Journal of Prolotherapy; VOLUME 3,2011 .

29. Maia MLM, Bonjardim LR, Quintans JSS, Ribeiro MAG, Maia LGM and Conti PCR. Effect of low-level laser therapy on pain levels in patients with temporomandibular disorders: a systematic review. J Appl Oral Sci 20:594602, 2012.

30. Honmura A, Ishii A, Yanase M, Obata J and Haruki E. Therapeutic effect of GaAlAs diode laser on experimentally induced inflammation in rats. Lasers Surg Med 13: 463469,1993 .
31. Mazzetto MO, Carrasco TG, Bidinelo EF, de Andrade Pizzo $\mathrm{RC}$, and Mazzetto RG. Low intensity laser application in temporomandibular disorders: a phase I double-blind study. Cranio 25(3):186-192,2007.

32. Nunez SC, Garcez AS, Suzuki SS and Riberio MS. Management of Mouth Opening in Patients with Temporomandibular Disorders through LowLevel Laser Therapy and Transcutaneous Electrical Neural Stimulation. Photomed and Laser surg 24(1):45-49, 2006.

33. Shukla D, Muthusekhar MR. Efficacy of low-level laser therapy in temporomandibular disorders: A systematic review. Natl J Maxillofac Surg.; 7:62-66. 2016.

34. Brochado FT, Jesus LH, Carrard VC, Freddo AL, Chaves KD, Martins MD. Comparative effectiveness of photobiomodulation and manual therapy alone or combined in TMD patients: a randomized clinical trial. Braz Oral Res. 10;32:e50 2018.

35. Sayed N, Murugavel C, Gnanam A.Management of Temporomandibular Disorders with Low Level Laser Therapy.J Maxillofac Oral Surg. ;13:444-50,2014. 